Results: Fifteen eyes (52\%) in group A achieved a visual acuity of 0.5 or better and $8(28 \%)$ achieved a visual acuity of 1.0, while 4 eyes in group B developed phthisis bulbi. For eyes with a preoperative visual acuity of hand motions or worse, there was no correlation between final visual acuity and preoperative visual acuity. The overall culture-positive rate was 57\%. In group A, methicillin-resistant Staphylococcus epidermidis was identified in 6 eyes, methicillinresistant Staphylococcus aureus (MRSA) in 3 eyes and enterococcus in 2 eyes. In group B, $\alpha$-hemolytic streptococcus (AHS) was identified in 4 eyes, aspergillus in 1 eye, and MRSA in 1 eye. All isolates were sensitive to vancomycin with the exception of the aspergillus. AHS infection appeared to be associated with wound failure from the initial cataract surgery and a poor visual outcome. In 3 of the eyes that developed phthisis bulbi, intravitreal injection of antibiotics was not performed.

Conclusion: Early vitrectomy and intravitreal injection of vancomycin may improve visual outcomes, but infection with AHS may be associated with cataract surgery wound failure and a poor visual outcome.

Nippon Ganka Gakkai Zasshi (J Jpn Ophthalmol Soc 107:590-596, 2003)

Kazuhiro Oshitari, Akito Hirakata, Annabelle A Okada, Tetsuo Hida, Hitoshi Oda, Daijiro Miki, Toshiyuki Nagamoto and Takaaki Fujiwara

Department of Ophthalmology, Kyorin University School of Medicine

DOI 10.1007/s10384-004-1020-8

\section{Serial Topographic Changes at the Optic Disc in Normal-tension Glaucoma Viewed with Scanning Laser Tomography}

Purpose: To evaluate topographic changes in normaltension glaucoma using serial optic disc images obtained by scanning laser tomography.

Subjects and Methods: Fifty-six eyes of 30 patients with normal-tension glaucoma were imaged using the Heidelberg Retina Tomograph (HRT) four or more times during follow-up periods of more than 3 years. Disc progression was determined by regression analysis of global and segmental changes in optic disc parameters. Visual field progression was determined by Humphrey Field Analyzer (HFA) program 30-2 and was compared with disc progression.

Results: The global parameters of the optic disc showed progression in 21 of 56 eyes $(37.5 \%)$. Progression was found in 30 eyes $(53.6 \%)$ at the superotemporal segment, in 24 eyes $(42.9 \%)$ at the temporal segment, in 27 eyes $(48.2 \%)$ at the inferotemporal segment, in 21 eyes $(37.5 \%)$ at the superonasal segment, in 23 eyes $(41.1 \%)$ at the nasal segment, and in 27 eyes $(48.2 \%)$ at the inferonasal segment. The visual field showed progression in 19 eyes $(33.9 \%)$. The progression in the optic disc parameters did not necessarily agree with that in the visual field.

Conclusions: Glaucomatous disc changes over time can be determined with HRT using regression analysis.

Nippon Ganka Gakkai Zasshi (J Jpn Ophthalmol Soc 107:597-601, 2003)

\begin{abstract}
Akiko Kato ${ }^{1}$, Kazuhisa Sugiyama ${ }^{1}$, Yoshiki Kono ${ }^{2}$, Hideya Uchida ${ }^{1}$, Goji Tomita ${ }^{3}$ and Tetsuya Yamamoto ${ }^{1}$

${ }^{1}$ Department of Ophthalmology, Gifu University School of Medicine; ${ }^{2}$ Department of Ophthalmology, Gifu Municipal Hospital; ${ }^{3}$ Department of Ophthalmology, The University of Tokyo Graduate School of Medicine
\end{abstract}

DOI 10.1007/s10384-004-1021-7

\section{Preferred Retinal Locus in Patients with Age-related Macular Degeneration}

Purpose: To evaluate the status and location of the preferred retinal locus (PRL) and visual function in age-related macular degeneration (AMD).

Subjects and Methods: Thirty-five patients were examined. They were diagnosed as having bilateral AMD in the disciform stage. Scanning laser ophthalmoscopic microperimetry was performed to detect the PRL. The retinal conditions of the PRL were classified using color fundus photographs and fluorescein angiography. The eccentricities of PRL from the fovea were measured. The relationships among visual acuity, retinal sensitivity, and the eccentricities of the PRL were evaluated.

Results: In 34 of the 35 eyes, PRLs had shifted from the fovea to the closest extrafoveal site, either an area of healthy retina or an atrophic lesion of the retinal pigment epithelium, whichever was closest to the fovea. In the eye in which the healthy retina was located distant from the fovea, the PRL had shifted to the atrophic pigment epithelium nearest the fovea. When it was possible for a PRL to move with similar eccentricity away from the fovea, it moved to the upper or temporal retina. The PRL eccentricities from the fovea ranged from 0.5 to 11 degrees, and the visual acuities ranged from 0.08 to 0.4 . There were differences in visual acuity even in eyes with the same eccentricity of PRLs, depending on the status of the retina in those eyes.

Conclusions: If there is scar formation in the fovea, the PRL moves to the nearest edge of the scar. Visual acuity and retinal sensitivity are determined by the status of the PRL. Nippon Ganka Gakkai Zasshi (J Jpn Ophthalmol Soc 107:602-606, 2003)

\section{Kyoko Fujita and Mitsuko Yuzawa}

Department of Ophthalmology, Surugadai Hospital of Nihon University

DOI 10.1007/s10384-004-1022-6 